\title{
Application of the amplification-free SERS-based CRISPR/Cas12a platform in the identification of SARS-CoV-2 from clinical samples
}

\author{
Jiajie Liang ${ }^{1,3+}{ }^{\text {, Peijun Teng }}{ }^{1+}$, Wei Xiao ${ }^{2 \dagger}$, Guanbo He ${ }^{3}$, Qifang Song ${ }^{1}$, Ying Zhang ${ }^{1}$, Bin Peng ${ }^{1}$, Gan Li ${ }^{1}$,
} Liangshan $\mathrm{Hu}^{2^{*}}$, Donglin $\mathrm{CaO}^{2^{*}}$ and Yong Tang ${ }^{1^{*}}$

\begin{abstract}
The control of contagious or refractory diseases requires early, rapid diagnostic assays that are simple, fast, and easyto-use. Here, easy-to-implement CRISPR/Cas12a-based diagnostic platform through Raman transducer generated by Raman enhancement effect, term as SERS-CRISPR (S-CRISPR), are described. The S-CRISPR uses high-activity noble metallic nanoscopic materials to increase the sensitivity in the detection of nucleic acids, without amplification. This amplification-free platform, which can be performed within 30-40 min of incubation time, is then used for detection of SARS-CoV-2 derived nucleic acids in RNA extracts obtained from nasopharyngeal swab specimens $(n=112)$. Compared with the quantitative reverse transcription polymerase chain reaction (RT-qPCR), the sensitivity and specificity of S-CRISPR reaches $87.50 \%$ and $100 \%$, respectively. In general, the S-CRISPR can rapidly identify the RNA of SARS-CoV-2 RNA without amplification and is a potential strategy for nucleic acid point of care test (POCT).
\end{abstract}

Keywords: Sliver nanoparticle, Surface-enhanced Raman scattering, COVID-19, SARS-CoV-2, CRISPR, Cas12a, Biosensors

\section{Introduction}

Contagious diseases including severe acute respiratory syndrome and refractory diseases such as prostatic cancer endanger public health and property [1]. In early January 2020, the coronavirus disease 2019 (COVID19), a cluster of cases with pneumonia, due to infection with the severe acute respiratory syndrome coronavirus

\footnotetext{
*Correspondence: liangshan8027@163.com; caodl@126.com; tyjaq7926@163.com

†Jiajie Liang, Peijun Teng and Wei Xiao contributed equally to this work

${ }^{1}$ Department of Bioengineering, Guangdong Province Engineering Research Center of Antibody Drug and Immunoassay, College of Life Science and Technology, Jinan University, Guangzhou 510632, People's Republic of China

2 Department of Laboratory Medicine, Guangdong Second Provincial General Hospital, Guangzhou 510317, People's Republic of China Full list of author information is available at the end of the article
}

2 (SARS-CoV-2), was first reported [2]. Soon afterward, this new coronavirus paralyzed the world because of the long incubation periods of patients and the absence of obvious biomarkers [3, 4]. Therefore, early, rapid, and easy-to-use diagnostic method is essential to identify patients and implement appropriate containment strategies, thereby preventing spread of the virus [5-7].

The RNA-guide endonuclease named clustered regularly interspaced short palindromic repeats (CRISPR)associated (Cas) enzyme, has been exploited as an efficient genome editing tool $[8,9]$. In addition to targeting endonuclease activity (targeted cleavage), CRISPR types III, V, and VI RNA-guided nucleases (Cas12, Cas13, and Cas14) display collateral target-activated, nonspecific single-stranded nucleic acid hydrolysis activity (collateral cleavage), which supports the current CRISPR/Cas-based original author(s) and the source, provide a link to the Creative Commons licence, and indicate if changes were made. The images or other third party material in this article are included in the article's Creative Commons licence, unless indicated otherwise in a credit line to the material. If material is not included in the article's Creative Commons licence and your intended use is not permitted by statutory regulation or exceeds the permitted use, you will need to obtain permission directly from the copyright holder. To view a copy of this licence, visit http://creativecommons.org/licenses/by/4.0/. The Creative Commons Public Domain Dedication waiver (http://creativeco mmons.org/publicdomain/zero/1.0/) applies to the data made available in this article, unless otherwise stated in a credit line to the data. 
nucleic acid diagnostic assays with advantages of reduced reaction time and amplification of self-signal [10-12]. Because of their specificity and the relatively simple design of guide RNAs, it is promising to utilize CRISPR/ Cas enzymes nucleic acids detection through fluorescent transduction systems or lateral-flow strips [13-15]. In combination with amplification of nucleic acid, it is suggested that CRISPR/Cas-based nucleic acid diagnostic approaches can be used to test various substances, including single nucleotide polymorphisms, miRNA, methyladenosine and pathogens [16-20]. Importantly, COVID-19, as a fulminant infectious disease, can also be detected using CRISPR/Cas-based nucleic acid diagnostic methods [5, 21-23]. Although the fluorescent signal is relatively sensitive, nucleic acid amplification remains a necessary step to improve sensitivity, resulting in complex experimental operation and primer design, and thus limiting their applications [24-26].

Clinically, the lower the detection limit, the easier the patient is to identify. Therefore, it is importance of low limit of detection for clinical SARS-CoV-2 early screening. For improving the sensitive, presently, electrochemical and gas- volumetric sensing strategies have been applied in CRISPR/Cas-based biosensors, which have shown good performance and achieved amplificationfree nucleic acid detection [27-30]. Noble metallic nanoscopic surfaces generate Raman enhancement effect, termed as surface-enhanced Raman scattering (SERS), an alternative high sensitive sensing strategy, also has potential application in CRISPR/Cas-based biosensors. This has resulted in the advancement of the use of SERSbased sensors [31, 32].

This study applied CRISPR/Cas12a-based methods in the development of SERS-based CRISPR diagnostic platform (S-CRISPR) because of their relative highsensitivity of the transduction systems compared with the fluorescent transducer. Further, we used S-CRISPR to detect nucleic acids without amplification, particularly in the detection of SARS-CoV-2 derived RNA from nasopharyngeal swab samples. The S-CRISPR achieves $87.50 \%$ sensitivity and $100 \%$ specificity compared with quantitative reverse transcription polymerase chain reaction (RT-qPCR). Our results show that the amplificationfree S-CRISPR has the potential for use as an early rapid screening diagnostic tool.

\section{Materials and methods Materials and reagents}

The silver nitrate $\left(\mathrm{AgNO}_{3}, 99.8 \%\right)$ was purchased from Sinoreagent (Shanghai, China). Tween-20 and Chloroauric acid $\left(\mathrm{HAuCl}_{4}\right)$ were obtained from Amresco (USA). Trisodium citrate and 4-aminothiophenol (4-ATP) were obtained from Sigma-Aldrich. Magnetic beads (MBs)@ Streptavidin was purchased from BEAVER (Suzhou, China). The RNase Inhibitor and the NEBuffer 2.1 were obtained from the New England Biolabs (USA). Microwell plate was obtained from CORNING, USA.

\section{Clinical samples collection and ethics statement}

The procedures used in this study for collection and treatment of clinical samples of COVID-19 (Characteristics of patients listed in Additional file 1: Table S1) were approved by the Scientific Research Ethics Review Committee of the Guangdong Second Provincial General Hospital (Ethical Approval No. 20200915-01-01-YXKXYJ-CRB) and were in accordance with the standard operation of WHO. Patients or their legal representatives had provided informed consent. Nasopharyngeal swab samples from patients with suspected SARS-CoV-2 infection were analysed at the Department of Laboratory Medicine, Guangdong Second Provincial General Hospital. RNA was extracted from the samples using the MAGPURE RNA Kit (Hybribio, Guangdong, China) according to the manufacturer's instructions. RNA extracts were initially used for molecular diagnosis of SARS-CoV-2 by RT-qPCR targeting N and orf1a genes using 2019-nCoV RT-qPCR Kit (DAAN Gene). Excess RNA extracts from these samples were used for results validation of S-CRISPR. Samples were randomized and validation assays were performed in a blinded manner.

\section{Nucleic acid preparation}

The N gene fragment from the SARS-CoV-2 (Wuhan-1 strain, GenBank: MN908947) was generated by Sangon Biotech (Shanghai, China) and was incorporated into the pUC57 vector. The Certificate of Analysis (COA) report from manufacturer indicated the concentration of plasmid was identified by Ultraviolet Absorption Spectrometry (A260) and the purity was identified by A260/ A280. The CRISPR-RNA (crRNA) of target sites from the genome of SARS-CoV-2 was designed by CHOPCHOP and Benchling. All other DNA and RNA used in this study were generated by the GenScript (Nanjing, China) and these sequences are listed in Additional file 1: Table S2. All RNAs were aliquoted and kept at $-80{ }^{\circ} \mathrm{C}$ for future experiments.

\section{Verification of the Cas12a-based assay using fluorescent} LbCas12a trans-cleavage assays were conducted as previous paper [28]. Target DNA was incubated with $50 \mathrm{nM}$ LbCas12a, $62.5 \mathrm{nM}$ crRNA, $40 \mathrm{U}$ RNase Inhibitor and $50 \mathrm{nM}$ linear ssDNA labeled with a quencher (ssDNAFQ) and a fluorophore in $1 \times$ NEBuffer 2.1 for $20 \mathrm{~min}$ at 
$37{ }^{\circ} \mathrm{C}$ and then distributed in 384-well plates for the florescent readout. To detect fluorescence, signal with excitation at $485 \mathrm{~nm}$ and emission at $535 \mathrm{~nm}$ was detected using the SYNERGY microplate reader (BioTek Instruments, $\mathrm{H} 1$ ).

For fluorescent Cas12a-based SARS-CoV-2 N gene plasmid detection, the plasmid was incubated with $50 \mathrm{nM}$ LbCas12a, $62.5 \mathrm{nM}$ crRNA, 40 U RNase Inhibitor and $50 \mathrm{nM}$ ssDNA-FQ in $1 \times$ NEBuffer 2.1 for $20 \mathrm{~min}$ at $37^{\circ} \mathrm{C}$ and then distributed in 384-well plates for the florescent readout. SYNERGY microplate reader (BioTek Instruments, $\mathrm{H1}$ ) with the excitation at $485 \mathrm{~nm}$ and emission at $535 \mathrm{~nm}$ was used for fluorescence detection.

\section{The construction of the portable Raman plate reader}

The Raman plate reader was assembled using laser-cut acrylic housing and readily available consumer components. The laser module was fixed on the top of the device with up and down adjustment allowing accurate focusing. The microwell plate was fixed to a magnetic plate holder, which can be inserted in the device to measure the Raman signal.

\section{The procedure of SERS-based CRISPR diagnostic platform}

To obtain SERS probe, we first prepared silver nanoparticle (AgNPs) according to those previously described [31]. Ultrapure water $(500 \mathrm{~mL})$ was used to dissolve $\mathrm{AgNO}_{3}$ (90 $\mathrm{mg}$ ) and the solution was boiled. To the boiling silver nitrate solution, $1 \%$ trisodium citrate aqueous solution $(10 \mathrm{~mL})$ was added dropwise and the solution was vigorously stirred. The mixed solution was boiled for additional $30 \mathrm{~min}$ and until a stable green-gray AgNPs is produced. The AgNPs solution $(10 \mathrm{~mL})$ was mixed with $10 \mu \mathrm{L}$ of $1 \mathrm{mM}$ 4-ATP was consistently stirred for $1 \mathrm{~h}$ at room temperature. The mixture was centrifuged at $6000 \mathrm{rpm}$ at $4{ }^{\circ} \mathrm{C}$ for $10 \mathrm{~min}$. The resulting pellet (AgNPs@4ATP) was suspended in $10 \mathrm{~mL}$ ultrapure water. Then, $100 \mu \mathrm{L}$ MBs@streptavidin were washed three times by magnetic shelf, and resuspended by $500 \mu \mathrm{L}$ Buffer 1 . $30 \mu \mathrm{L} 4 \mathrm{mM}$ SH-ssDNA-biotin was added to the MBs@ streptavidin and incubated $30 \mathrm{~min}$. The MBs-ssDNASH were reacted with AgNPs@4ATP for 30 min to form SERS probe. The SERS probe was washed three times and was stored at $4{ }^{\circ} \mathrm{C}$ till used.

The gene target of SARS-CoV-2 RNA was reverse transcribed by ReverTra Ace ${ }^{\circledR}$ qPCR RT Kit (TOYOBO, FSQ101) following instructions of the manufacturer. The reverse transcribed product of SARS-CoV-2 RNA gene target was incubated with $50 \mathrm{nM}$ LbCas12a, $62.5 \mathrm{nM}$ crRNA, $40 \mathrm{U}$ RNase Inhibitor and SERS probe in $1 \times$ NEBuffer 2.1 at $37^{\circ} \mathrm{C}$ for $20 \mathrm{~min}$. The MBs were washed three times by magnetic shelf and resuspended by $100 \mu \mathrm{L}$

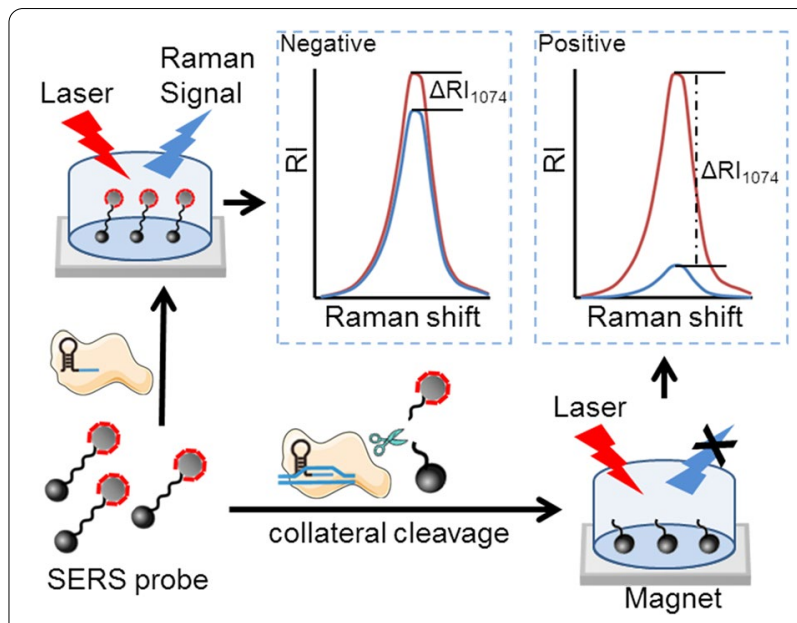

Fig. 1 Schematic of the S-CRISPR assay

ultrapure water. The well plates placed in portable Raman plate reader and the Raman signal was measured one by one using a portable Raman spectrometer (QSPEC, SmartRaman) with $300 \mathrm{~mW}$ power of excitation laser at $785 \mathrm{~nm}$ and accumulation time of $10 \mathrm{~s}$. The characteristic Raman peak $\left(1074 \mathrm{~cm}^{-1}\right)$ of 4-ATP was noticeably identified and was used for analysis.

\section{S-CRISPR for the detection of SARS-CoV-2 from clinical samples}

RNA extracts from nasopharyngeal swabs were reverse transcribe by ReverTra Ace ${ }^{\circledR} \mathrm{qPCR}$ RT Kit (TOYOBO, FSQ-101) following instructions of the manufacturer. The reverse transcribed products were detected by S-CRISPR respectively according to the procedure of S-CRISPR.

\section{Statistical analysis}

Data were analysed with the GraphPad Prism 8. All results were presented as mean \pm standard error unless stated otherwise. The Clopper-Pearson method was used to calculate two-sided confidence intervals of specificity, sensitivity, positive predictive agreement (PPA) and negative predictive agreement (NPA).

\section{Results and discussions}

Verification of the Cas12a-based assay for SARS-CoV-2 RNA detection

CRISPR-Cas12a proteins are RNA-guided enzymes that can bind and cleave double-stranded DNA (dsDNA) or single-stranded DNA (ssDNA) using a RuvC catalytic pocket $[33,34]$. Once the Cas12a-crRNA duplex recognizes the target DNA, Cas12a can activate its singlestranded deoxyribonuclease activity, in addition to its endonuclease activity, to nonspecifically cleave nearby 

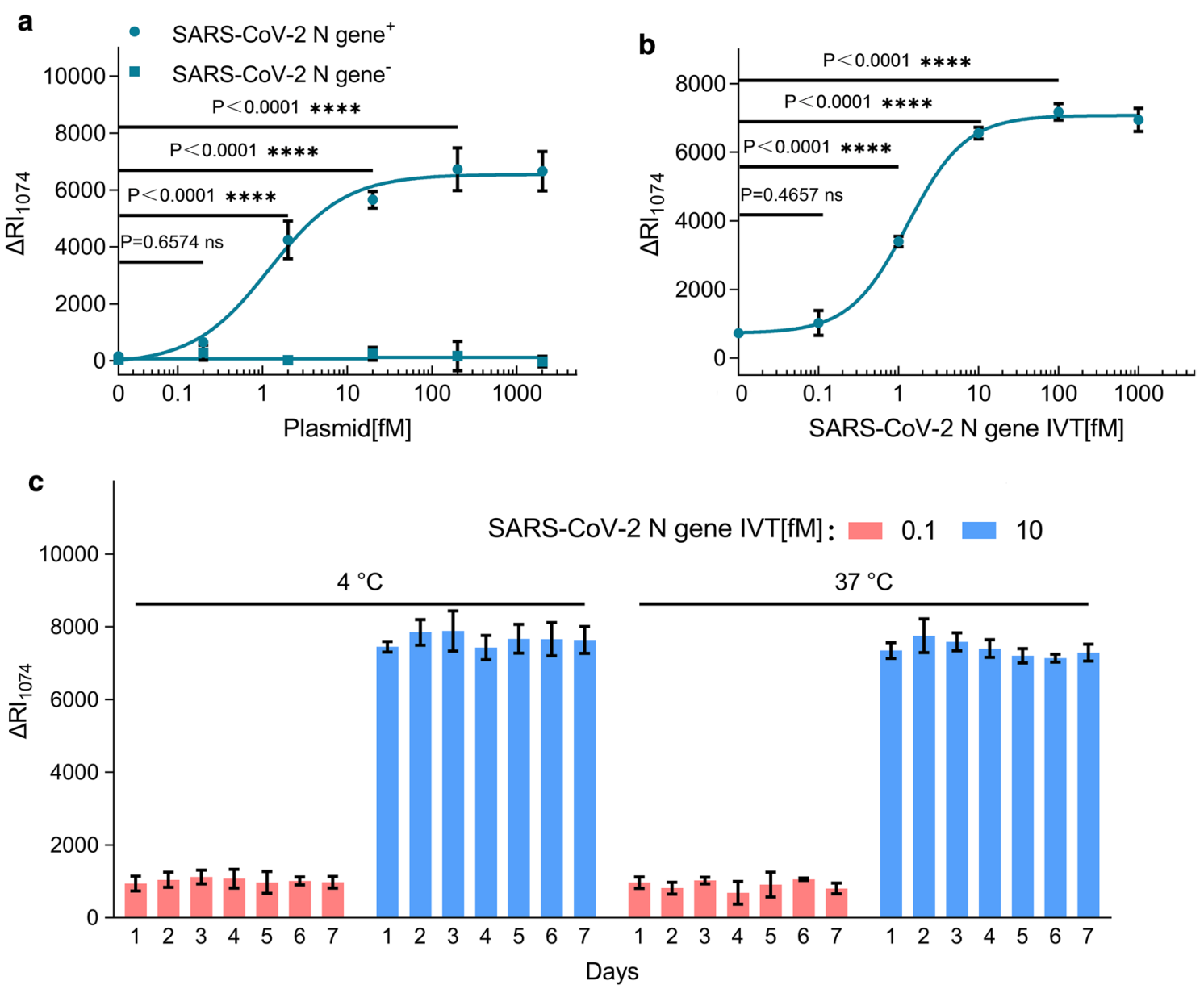

Fig. 2 S-CRISPR for SARS-CoV-2 detection. a Titration of SARS-COV-2 N gene-positive and -negative plasmids as detected by the S-CRISPR. The fitting model: [Inhibitor] vs. response-Variable slope (four parameters). Equation: $Y=6548+(-111.1-6548) /\left(1+(1.204 / X)^{-0.9674}\right), R^{2}=0.9665$. b S-CRISPR was assessed using IVT RNA products from SARS-CoV-2. The fitting model: [Inhibitor] vs. response-Variable slope (four parameters). Equation: $Y=7074+(724.2-7074) /\left(1+(1.304 / X)^{-1.201}\right), R^{2}=0.9937$. $\mathbf{c}$ Initial validation of the storage life of the S-CRISPR assay. The components of the S-CRISPR assay were stored in $4^{\circ} \mathrm{C}$ and $37^{\circ} \mathrm{C}$ for up to 7 days, and the IVT RNA products $(0.1 \mathrm{fM}, 10 \mathrm{fM})$ were used to assess the signal change during the indicated storage period. The LoD was defined by a significant variation of $\triangle$ Raman signal at the lowest concentration. Standard deviations were determined based on three independent experiments

ssDNAs, which is termed as trans-cleavage [35]. This high-efficiency trans-cleavage activity (approximately 1250 turnovers per second) and the crRNA-dependent sequence specificity of Cas12a have been used in the identification of nucleic acids with high specificity, sensitivity and rapidity [11]. Almost all of these assays utilized activated Cas12a to cleave the ssDNA-FQ which can then release a fluorescence signal (Additional file 1: Figure S1a).

Here, we first validated the collateral cleavage activity of Cas12a-crRNA duplex. The ssDNA-FQ was cleaved by Cas12a in response to the verified fragments of dsDNA triggers (Target DNA) confirmed the performance of the cognate crRNA (Additional file 1: Figure S1b). Cas12a with cognate crRNA detected its Target DNA at the lowest concentration of $0.01 \mathrm{nM}$ (Additional file 1: Figure $\mathrm{S} 2 \mathrm{a}-\mathrm{c}$ ) according to fluorescence readout and optimized reaction conditions (the concentration of crRNA was $62.5 \mathrm{nM}$ and Cas12a was $50 \mathrm{nM}$ ) were selected for subsequent experiments (Additional file 1: Figure S2d). The time-course curves indicated that reaction acceleration in the first $20 \mathrm{~min}$ and complete reaction after $20 \mathrm{~min}$, Because the point-in-time of $20 \mathrm{~min}$ was the smallest point-in-time which the fluorescence ratio of adjacent points-in-time that nearest $1(<0.99)$. Comparing the different rates of each point-in-time, $20 \mathrm{~min}$ was chosen as reaction time of trans-cleavage. 


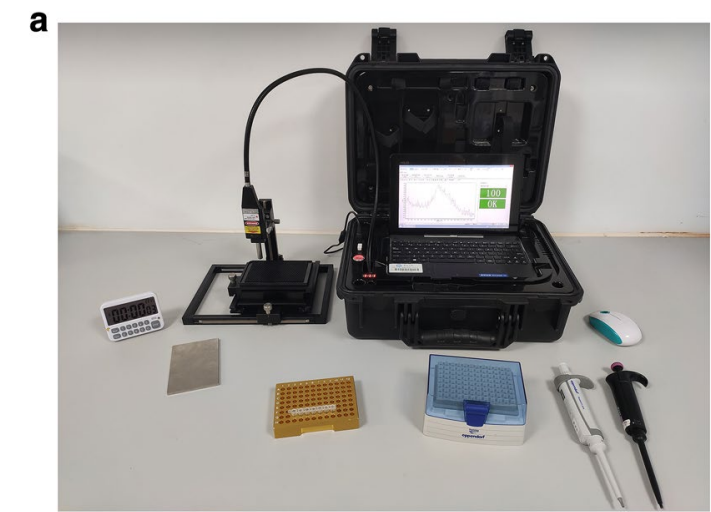

b

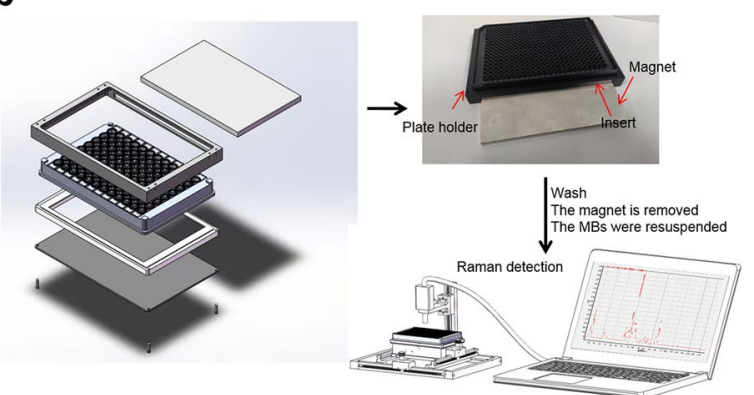

Fig. 3 Portable Raman plate reader. a Schematic diagram of the low-cost, easy-to-use portable Raman plate reader developed to read the Raman output from a portable Raman spectrometer. b operation procedure of the portable Raman plate reader

Following this, we designed crRNA for the rapid and specific detection of SARS-CoV-2 (accession NC_045512) in the N gene (Additional file 1: Figure S3a) following the World Health Organization (WHO) guidelines (January 17, 2020). Using plasmids encoding the $\mathrm{N}$ gene sequence of SARS-CoV-2, SARS-CoV (Accession NC_004718) and bat SARS-like CoV (Accession MG772933), we showed that the CRISPR-Cas12-based detection assay could distinguish SARS-CoV-2 from the other related coronavirus strains with no cross-reactivity (Additional file 1: Figure S3b, c). Thus, we next explored the ability of SERS-based CRISPR diagnostic platform to identify amplification-free SARS-CoV-2 RNA.

\section{Establishment of SERS-based CRISPR diagnostic platform for SARS-CoV-2 RNA detection}

This study utilized SERS transduction for CRISPR/ Cas12a-based method to identify SARS-CoV-2 RNA. Based on the findings of previous studies [31], AgNP@4ATP was used as a Raman tag and linked to MB through an ssDNA form a SERS probe. The target DNA activates the Cas12a collateral cleavage, thus removing the Raman tags off the MBs and decreasing the Raman intensity. The Raman intensity of negative quality control test is represented by red line and sample test is represented by blue line $\left(\Delta R I=R I_{\text {control test }}-R_{\text {sample }}\right.$ test) (Fig. 1). We first prepared the SERS probe linked to AgNPs@4ATP and MBs@streptavidin through $\mathrm{SH}$-ssDNA-biotin (Additional file 1: Figure S4a) and identified the concentrations of AgNP@4ATP and SHssDNA-biotin (Additional file 1: Figure S4b). Subsequently, the Target DNA was employed to verify that the SERS probe can be cleaved by Cas12a to change the SERS intensity (Additional file 1: Figure S4c). Importantly, the lowest detection limit was $1 \mathrm{fM}$, indicating the potential of the S-CRISPR assay to detect SARS-CoV-2 RNA.

Next, we focused on developing the S-CRISPR for the SARS-CoV-2 detection. The SARS-CoV-2 N gene-positive plasmid was used for the verification of S-CRISPR. A curve for performance was constructed using six dilutions of an $\mathrm{N}$ gene-positive and -negative plasmid, with three replicates at each dilution (Fig. 2a). We confirmed the ability of the assay to generate a significant variation of $\Delta$ Raman signal at the lowest concentration of $\mathrm{N}$ gene-positive plasmid, and the lowest detection limit was $2 \mathrm{fM}$, indicating the feasibility of the S-CRISPR assay to detect the cDNA reverse transcribed from SARS-CoV-2 RNA. Subsequently, a synthetic, in vitro-transcribed (IVT) SARS-CoV-2 RNA gene target was used to mimic the detection of RNA viruses. A standard curve for quantitation was constructed using six dilutions of a control IVT viral nucleoprotein RNA (Fig. 2b). The analytic limit of detection (LoD) defined by a significant variation of $\Delta$ Raman signal at the lowest concentration was determined to be $1 \mathrm{fM}$. Additionally, the initial validation of the stability of the SERS probe and the Cas12acrRNA duplex indicated no noticeable changes in the performance of the S-CRISPR assay to detect the RNA of SARS-CoV-2 up to day 7 at room temperature and $37{ }^{\circ} \mathrm{C}$ (Fig. 2c). Therefore, these findings suggest that the proposed S-CRISPR can detect SARS-CoV-2.

\section{Development of portable Raman plate reader for SERS-based CRISPR diagnostic platform}

The implementation of S-CRISPR generally requires large Raman spectrometers which may increase the assay expense and reduce the portability for application in clinical diagnosis. Currently, although portable Raman spectrometers are becoming commercially available, they lack accuracy and stability because of the large phase difference and low spatial resolution and integration 


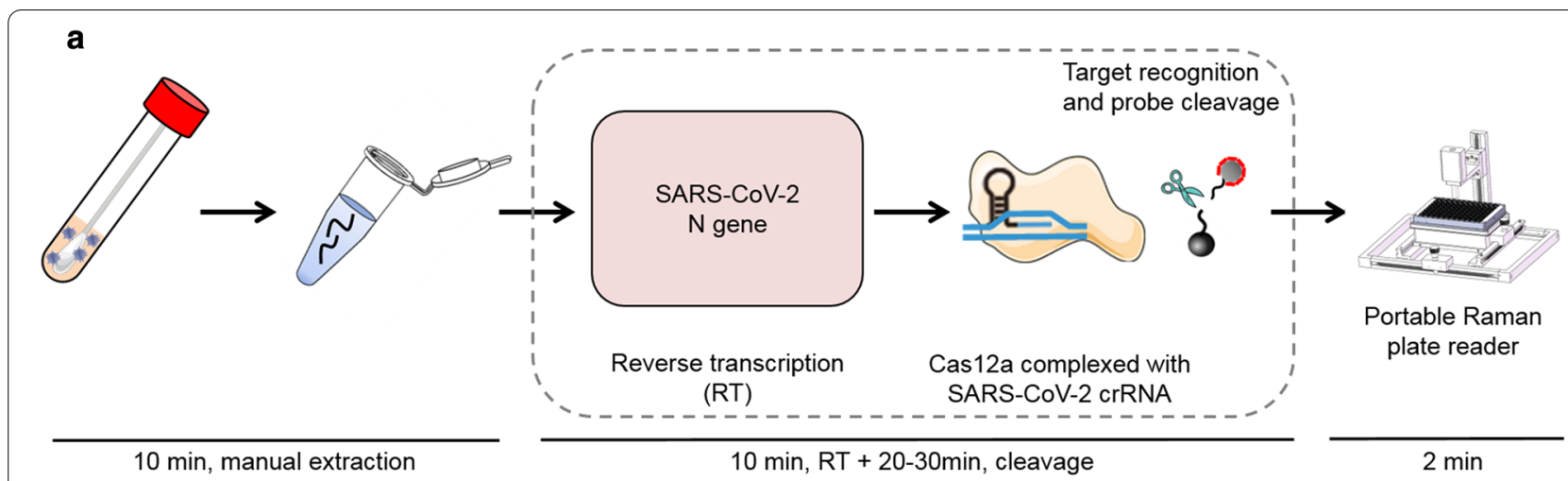

b

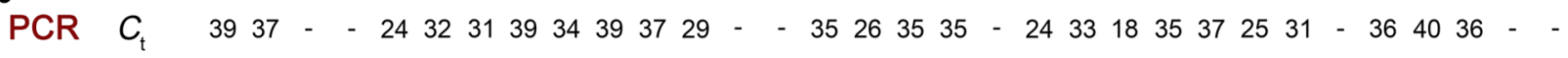

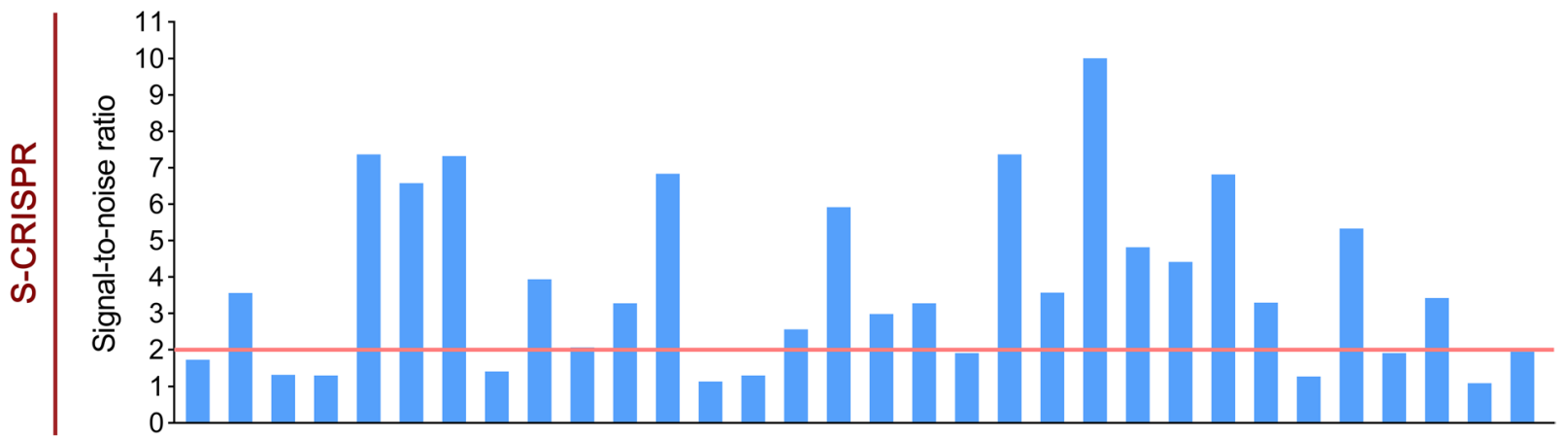

Sample No. $1 \quad 2 \quad 3 \quad 44 \quad 5 \quad 6 \quad 7 \quad 8 \quad 9 \quad 1011121314151617181920212223242526272829303132$

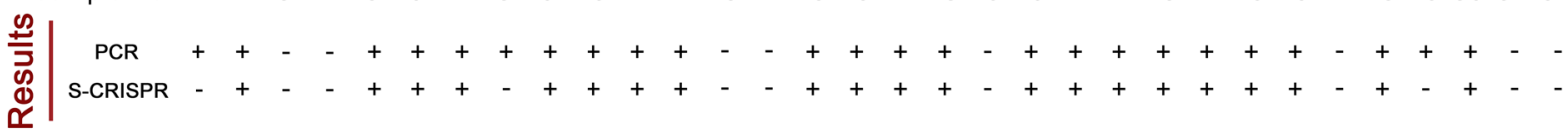

C

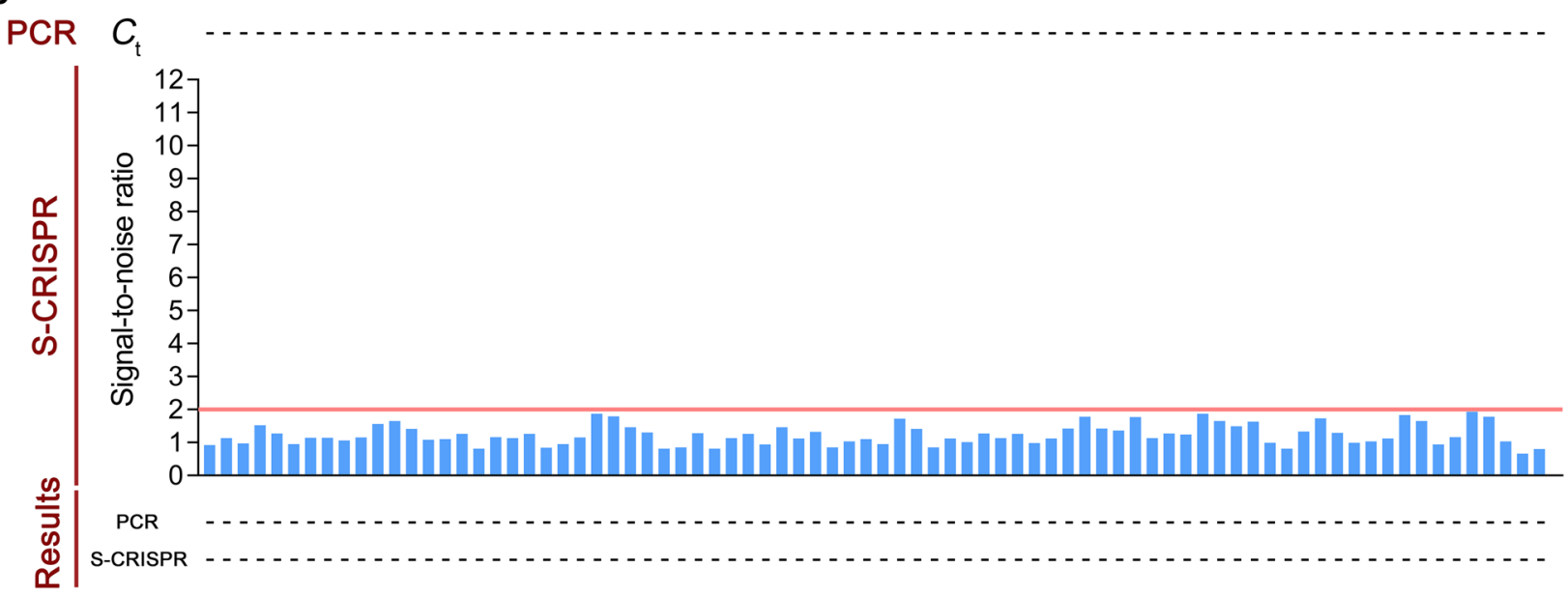

Fig. 4 S-CRISPR for the identification of SARS-CoV-2 from nasopharyngeal swab samples. a Schematic of the workflow for the SARS-CoV-2 S-CRISPR. The traditional RNA extraction protocol can be used as an initial step in CRISPR-based assays and is measured using portable Raman plate reader. $\mathbf{b}$ S-CRISPR for the identification of SARS-CoV-2 in 32 clinically diagnosed COVID-19 positive samples. The 80 negative samples are shown in c For the $\triangle \mathrm{RI}$ readouts, the noise is defined as the signal intensity of a negative sample (water) measured in parallel for a positive result, the threshold (pink line) of signal-to-noise ratio $(\mathrm{S} / \mathrm{N})$ is set at 2 
Table 1 Concordance between the results of the RT-qPCR and $\mathrm{S}$-CRISPR for the SARS-CoV-2 $\mathrm{N}$ gene detection

\begin{tabular}{lccc}
\hline & \multicolumn{3}{l}{ Samples diagnosed using RT-qPCR } \\
\cline { 2 - 4 } & Positive & Negative & Total \\
\hline S-CRISPR & & 0 & \\
Positive & 21 & 88 & 21 \\
Negative & 3 & 88 & 91 \\
Total & 24 & & 112 \\
\hline
\end{tabular}

(Additional file 1: Figure S5a, b). Here, we designed a portable Raman plate reader to provide robust and quantitative measurements of Raman signals with low cost (Fig. 3a). The Raman plate reader has a total cost of approximately $¥ 200$. Once the cleavage was completed in the microwell, the custom magnet was inserted in the plate holder and the free Raman tags were washed off. After washing, the custom magnet was removed from the plate holder, and the MBs were resuspended. At this point, the plate holder can be placed into the device, and the position of the laser module can be adjusted (Fig. 3b). As a proof of concept, we evaluated the DNase cleaving activity of the SERS probe and observed significant reads $(P<0.01)$ from the different concentrations of DNase with 10 replicates per concentration (Additional file 1: Figure S5c). The result indicated the stability of the portable Raman plate reader is suitable for the proposed S-CRISPR for the identification of SARS-CoV-2 from clinical samples.

\section{The application of SERS-based CRISPR diagnostic platform} in the detection of SARS-CoV-2 from clinical samples Lastly, we validated the feasibility of the S-CRISPR for SARS-CoV-2 detection from clinical samples. The S-CRISPR workflows were validated using 112 nasopharyngeal swab samples collected from patients in the Guangdong Second Provincial General Hospital (Fig. 4a). Of the 112 patients, 32 were COVID-19-positive based on clinical diagnosis and 80 were negative (patient characteristics are listed in Additional file 1: Table S1). Because RT-qPCR was performed before the proposed CRISPR-based assays for all samples, we kept the RNA samples at $-80{ }^{\circ} \mathrm{C}$ to reduce sample degradation and performed the CRISPR-based validation experiments in the Guangdong Second Provincial General Hospital to prevent sample degradation during transportation. Among the 112 nasopharyngeal swab samples, 21 samples were identified as positive (Fig. 4b) and 91 samples were identified as negative (Fig. 4c) by $\mathrm{N}$ gene S-CRISPR. Compared with RT-qPCR (Table 1), the S-CRISPR was $87.5 \%$ sensitive and $100 \%$ specific. These corresponded to $100 \%$ positive predictive agreement and $96.7 \%$ negative predictive agreement for the S-CRISPR (Table 2). Compared with that of other amplification-based CRISPR assays, our proposed assay was rapid (30-40 min) and easy-to-use, which are desirable properties for early rapid screening. In addition, our S-CRISPR used similar sample collection procedures and RNA extraction methods as that in RT-qPCR, which are easy to operate. The short time spent in the development and validation of the assays, i.e., < 2 weeks, during the detection of SARS$\mathrm{CoV}-2$ shows that these assays can offer rapid diagnosis of infections due to emerging viruses.

\section{Conclusions}

The global COVID-19 pandemic highlights the need for rapid, economical, and easy-to-use diagnostic kits, which would contribute to early decisions on disease-control strategies and management of patient treatments. In this study, utilizing the high-sensitivity of SERS, we developed amplification-free SERS-based CRISPR diagnostic platform for SARS-CoV-2 detection which could save the time of nucleic acid amplification. In addition, to advance the S-CRISPR to be used in diagnostics, we designed a portable Raman plate reader to measure Raman signals with advantages of high accessibility and low-cost. The S-CRISPR was able to detect SARS-CoV-2 genes from nasopharyngeal swab samples, in the absence of amplification, thereby serving as alternatives to conventional assays.

Overall, we validated the potential of S-CRISPR for nucleic acid detection. The S-CRISPR should be further developed to detect other genes of the SARS-CoV-2 genome and thus increase their sensitivity. The automation of the portable Raman plate reader and the stability of S-CRISPR should also be explored by future studies.

Table 2 Results for the sensitivity, specificity, and predictive agreement characterizations ${ }^{\mathrm{a}}$ of the S-CRISPR for the identification of the SARS-CoV-2 N gene

\begin{tabular}{lllll}
\hline & Sensitivity $[\mathbf{9 5} \% \mathrm{Cl}]$ & Specificity $[\mathbf{9 5 \%} \mathrm{Cl}]$ & PPA [95\% CI] & NPA [95\% Cl] \\
\hline S-CRISPR & $87.50 \%(66.54-96.72)$ & $100 \%(94.79-100)$ & $100 \%(80.76-100)$ & $96.70 \%(89.99-99.15)$ \\
\hline
\end{tabular}

a Values were calculated from 112 samples for clinical validation and compared with those of RT-qPCR

$\mathrm{Cl}$ confidence interval 


\section{Supplementary Information}

The online version contains supplementary material available at https://doi. org/10.1186/s12951-021-01021-0.

Additional file 1: Figure S1. Verification of the collateral cleavage activity of Cas12a using fluorescent assay. (a) Schematic of the Cas12a-based fluorescent assay; (b) The collateral cleavage of the Cas12a-crRNA duplex activated by dsDNA triggers. Randomly designed TargetDNAs activate respectiveCas12a-crRNA duplex. Figure S2. Confirmation of the Target DNA homologous crRNA using fluorescent assay and the optimized conditions. (a) Cas12a crRNA 1 was programmed to specifically Target DNA 1 , and its time-course detection. The numbers represent the fluorescence ratio of adjacent points-in-time;(b) Cas12a crRNA 2 was programmed to specifically Target DNA 2;(c) Cas12a crRNA 3 was programmed to specifically Target DNA 3;(d) The concentrations of crRNAs and Cas12a were optimized. All the error bars are determined from three independent experiments. Figure S3. Confirmation of SARS-CoV-2 homologous crRNA. (a) Genome map of the SARS-CoV-2 showing crRNA. Visualization of the crRNA to identify N gene region in the SARS-CoV-2 genome; (b) crRNA specificity. Cas12a crRNA is programmed to specifically target SARS-CoV-2. The $\mathrm{N}$ gene crRNA used in the assay was specific for SARS-CoV-2 and failed to detect SARS-CoV and bat SARS-like coronavirus; (c) Time-course detection of the plasmids $(2 \mathrm{nM}$ ) containing the $\mathrm{N}$ gene sequence of SARS-CoV-2, SARS-CoV and bat SARS-like coronavirus. The numbers represent the fluorescence ratio of adjacent points-in-time. All the error bars are determined from three independent experiments. Figure S4. Confirmation of the S-CRISPR assay. (a) SEM images (ZEISS ULTRA 55 field emission scanning electron microscopy) of AgNPs (l; scale bar: $200 \mathrm{~nm}$; $30 \mathrm{~K} \times$ ), SERS probe (MBs-ssDNA-AgNPs; ll; scale bar: $500 \mathrm{~nm} ; 20 \mathrm{~K} \times$ ), and SERS probe cleaved by Cas12a (III; scale bar: $500 \mathrm{~nm} ; 20 \mathrm{~K} \times$ ). AgNPs were successfully linked on the surfaces of the MBs and unlinked from them after cleavage; (b) The concentrations of SH-ssDNA-biotin and AgNPs@4ATP are optimized; (c) S-CRISPR assay was confirmed using Target DNA 1. All the error bars are determined from three independent experiments. Figure S5. Portable Raman plate reader. (a) The portable Raman spectrometer (QSPEC, SmartRaman); (b) Manualoperation procedureof the portable Raman spectrometer; (c) DNase-cleaving SERS probe and signals as detected by the portable Raman plate reader. All the error bars are determined from ten independent experiments. Table S1. Characteristics of clinical samples from COVID-19-suspected patients. Table S2. Nucleic acids used in this study.

\section{Acknowledgements}

This work was supported by grants from the Natural Science Foundation of Guangdong Province, China (Grant No. 2021A1515010174), Research and Development and Popularization and Application of the Technology of Special Financial Foundation of Guangdong, China (Grant No. 2013-401), Doctoral Workstation Foundation of Guangdong Second Provincial General Hospital (Grant No. 2020BSGZ039), and The science foundation of Guangdong Second Provincial General hospital (Grant No. 3D-A2021003).

\section{Authors' contributions}

$J \mathrm{~L}, \mathrm{WX}$ and PT contributed equally to this work. JL and $\mathrm{YT}$ designed the study. $J \mathrm{~L}, \mathrm{WX}$ and PT, designed and performed experiments with the assistance of $\mathrm{BP}, \mathrm{YZ}$ and $\mathrm{GL} \mathrm{J}$ and $\mathrm{GH}$ designed the portable device. QS provided critical materials and reagents for the experiments. WX, LH and DC provided and facilitated testing of clinical samples. LH, DC and YT supervised the study. JL, WX and PT contributed to the writing of the manuscript and interpretation of data. All authors read and approved the final manuscript.

\section{Availability of data and materials}

All data generated or analyzed during this study are included in the article and additional file.

\section{Declarations}

\section{Ethics approval and consent to participate}

The procedures used in this study for collection and treatment of clinical samples of COVID-19 (Characteristics of patients listed in Additional file 1: Table S1) were approved by the Scientific Research Ethics Review Committee of the Guangdong Second Provincial General Hospital (Ethical Approval No. 20200915-01-01-YXKXYJ-CRB) and were in accordance with the standard operation of WHO.

\section{Consent for publication}

All authors have provided consent for the manuscript to be published.

\section{Competing interests}

The authors declare no competing fnancial interest.

\section{Author details}

${ }^{1}$ Department of Bioengineering, Guangdong Province Engineering Research Center of Antibody Drug and Immunoassay, College of Life Science and Technology, Jinan University, Guangzhou 510632, People's Republic of China.

${ }^{2}$ Department of Laboratory Medicine, Guangdong Second Provincial General Hospital, Guangzhou 510317, People's Republic of China. ${ }^{3}$ Guangdong Biowings Tech Limited, Foshan 528000, People's Republic of China.

Received: 13 July 2021 Accepted: 31 August 2021

Published online: 08 September 2021

\section{References}

1. Wang C, Horby PW, Hayden FG, Gao GF. A novel coronavirus outbreak of global health concern. Lancet. 2020;395:470-3.

2. Zhu N, Zhang DY, Wang WL, Li XW, Yang B, Song JD, et al. A novel coronavirus from patients with pneumonia in China, 2019. N Engl J Med. 2020:382:727-33.

3. Bai Y, Yao LS, Wei T, Tian F, Jin DY, Chen LJ, et al. Presumed asymptomatic carrier transmission of COVID-19. JAMA. 2020;323:1406-7.

4. Rothe C, Schunk M, Sothmann P, Bretzel G, Froeschl G, Wallrauch C, et al. Transmission of 2019-nCoV infection from an asymptomatic contact in Germany. N Engl J Med. 2020;382:970-1.

5. Patchsung M, Jantarug K, Pattama A, Aphicho K, Suraritdechachai S, Meesawat P, et al. Clinical validation of a Cas13-based assay for the detection of SARS-CoV-2 RNA. Nat Biomed Eng. 2020:4:1140-9.

6. Rajapaksha P, Elbourne A, Gangadoo S, Brown R, Cozzolino D, Chapman J. A review of methods for the detection of pathogenic microorganisms. Analyst. 2019:144:396-411.

7. Yüce M, Filiztekin E, Özkaya KG. COVID-19 diagnosis—a review of current methods. Biosens Bioelectron. 2021:172:112752.

8. Xu L, Wang J, Liu YL, Xie LF, Su B, Mou DL, et al. CRISPR-edited stem cells in a patient with HIV and acute lymphocytic leukemia. N Engl J Med. 2019;381:1240-7.

9. Lu Y, Xue JX, Deng T, Zhou XJ, Yu K, Deng L, et al. Safety and feasibility of CRISPR-edited T cells in patients with refractory non-small-cell lung cancer. Nat Med. 2020;26:732-40.

10. East-Seletsky A, O'Connell MR, Knight SC, Burstein D, Cate JHD, Tjian R, et al. Two distinct RNase activities of CRISPR-C2C2 enable guide-RNA processing and RNA detection. Nature. 2016;538:270-3.

11. Chen JS, Ma EB, Harrington LB, Da Costa M, Tian XR, Palefsky JM, et al. CRISPR-Cas12a target binding unleashes indiscriminate single-stranded DNase activity. Science. 2018;360:436-9.

12. Harrington LB, Burstein D, Chen JS, Paez-Espino D, Ma E, Witte IP, et al. Programmed DNA destruction by miniature CRISPR-Cas 14 enzymes. Science. 2018:362:839-42.

13. Gootenberg JS, Abudayyeh OO, Kellner MJ, Joung J, Collins JJ, Zhang F. Multiplexed and portable nucleic acid detection platform with Cas13, Cas12a, and Csm6. Science. 2018;360:439-44.

14. Ali Z, Aman R, Mahas A, Rao GS, Tehseen M, Marsic T, et al. iSCAN: an RT-LAMP-coupled CRISPR-Cas 12 module for rapid, sensitive detection of SARS-CoV-2. Virus Res. 2020;288:198129. 
15. Joung J, Ladha A, Saito M, Kim NG, Woolley AE, Segel M, et al. Detection of SARS-COV-2 with SHERLOCK One-Pot Testing. N Engl J Med. 2020;383:1492-4

16. Myhrvold C, Freije CA, Gootenberg JS, Abudayyeh OO, Metsky HC, Durbin AF, et al. Field-deployable viral diagnostics using CRISPR-Cas13. Science. 2018;360:444-8.

17. Ai JW, Zhou X, Xu T, Yang ML, Chen YY, He GQ, et al. CRISPR-based rapid and ultra-sensitive diagnostic test for Mycobacterium tuberculosis. Emerg Microbes Infect. 2019:8:1361-9.

18. Abudayyeh OO, Gootenberg JS, Essletzbichler P, Han S, Joung J, Belanto $\mathrm{J}$, et al. RNA targeting with CRISPR-Cas13. Nature. 2017;550:280-4.

19. Gootenberg JS, Abudayyeh OO, Lee JW, Essletzbichler P, Dy AJ, Joung $\mathrm{J}$, et al. Nucleic acid detection with CRISPR-Cas13a/C2c2. Science. 2017;356:438-42.

20. Chen Y, Yang SX, Peng S, Li W, Wu F, Yao Q, et al. N1-Methyladenosine detection with CRISPR-Cas13a/C2c2. Chem Sci. 2019;10:2959-75.

21. Broughton JP, Deng XD, Yu GX, Fasching CL, Servellita V, Singh J, et al. CRISPR-Cas12-based detection of SARS-CoV-2. Nat Biotechnol. 2020;38:870-4.

22. Ma PX, Meng QZ, Sun BQ, Zhao B, Dang L, Zhong MT, et al. MeCas12a, a highly sensitive and specific system for COVID-19 detection. Adv Sci. 2020;7:2001300.

23. Chen Y, Shi Y, Chen Y, Yang Z, Wu H, Zhou Z, et al. Contamination-free visual detection of SARS-CoV-2 with CRISPR/Cas12a: a promising method in the point-of-care detection. Biosens Bioelectron. 2020;169:112642.

24. Wu H, Qian C, Wu C, Wang Z, Wang D, Ye Z, et al. End-point dual specific detection of nucleic acids using CRISPR/Cas12a based portable biosensor. Biosens Bioelectron. 2020;157:112153.

25. Wang R, Qian C, Pang Y, Li M, Yang Y, Ma H, et al. opvCRISPR: one-pot visual RT-LAMP-CRISPR platform for SARS-Cov-2 detection. Biosens Bioelectron. 2021:172:112766.

26. Fozouni P, Son S, de León Derby MD, Knott GJ, Gray CN, D’Ambrosio MV, et al. Amplification-free detection of SARS-CoV-2 with CRISPR-Cas13a and mobile phone microscopy. Cell. 2020;184:323-33.

27. Bruch R, Baaske J, Chatelle C, Meirich M, Madlener S, Weber W, et al. CRISPR/Cas13a-powered electrochemical microfluidic biosensor for nucleic acid amplification-free miRNA diagnostics. Adv Mater. 2019;31:e1905311.

28. Shao N, Han X, Song YN, Zhang PC, Qin LD. CRISPR-Cas12a coupled with platinum nanoreporter for visual quantification of SNVs on a volumetric bar-chart chip. Anal Chem. 2019;91:12384-91.

29. Bruch R, Johnston M, Kling A, Mattmüller T, Baaske J, Partel S, et al. CRISPR-powered electrochemical microfluidic multiplexed biosensor for target amplification-free miRNA diagnostics. Biosens Bioelectron. 2021:177:112887

30. Xu W, Jin T, Dai Y, Liu CC. Surpassing the detection limit and accuracy of the electrochemical DNA sensor through the application of CRISPR Cas systems. Biosens Bioelectron. 2020;155:112100.

31. Liang JJ, Liu HW, Lan CF, Fu QQ, Huang CH, Luo Z, et al. Silver nanoparticle enhanced Raman scattering-based lateral flow immunoassays for ultra-sensitive detection of the heavy metal chromium. Nanotechnology. 2014;25:495501.

32. Liang JJ, Liu HW, Huang CH, Yao CZ, Fu QQ, Li XQ, et al. Aggregated silver nanoparticles based surface-enhanced Raman scattering enzyme-linked immunosorbent assay for ultrasensitive detection of protein biomarkers and small molecules. Anal Chem. 2015:87:5790-6.

33. Liang MD, Li ZL, Wang WS, Liu JK, Liu LS, Zhu GL, et al. A CRISPR-Cas12aderived biosensing platform for the highly sensitive detection of diverse small molecules. Nat Commun. 2019;10:3672.

34. Qian C, Wang R, Wu H, Zhang F, Wu J, Wang L. Uracil-mediated new photospacer-adjacent motif of Cas12a to realize visualized DNA detection at the single-copy level free from contamination. Anal Chem. 2019;91:11362-6.

35. Zuo XL, Fan CH, Chen HY. BIOSENSING: CRISPR-powered diagnostics. Nat Biomed Eng. 2017;1:1-2.

\section{Publisher's Note}

Springer Nature remains neutral with regard to jurisdictional claims in published maps and institutional affiliations.
Ready to submit your research? Choose BMC and benefit from:

- fast, convenient online submission

- thorough peer review by experienced researchers in your field

- rapid publication on acceptance

- support for research data, including large and complex data types

- gold Open Access which fosters wider collaboration and increased citations

- maximum visibility for your research: over $100 \mathrm{M}$ website views per year

At $\mathrm{BMC}$, research is always in progress.

Learn more biomedcentral.com/submissions 